Case Report

\title{
Rhabdomyolysis and Dengue Fever: A Case Report and Literature Review
}

\author{
Tanya Sargeant, ${ }^{1}$ Tricia Harris, ${ }^{1}$ Rohan Wilks, ${ }^{1}$ Sydney Barned, ${ }^{1}$ \\ Karen Galloway-Blake, ${ }^{1}$ and Trevor Ferguson ${ }^{1,2}$ \\ ${ }^{1}$ Department of Medicine, The University of the West Indies, Mona, Kingston 7, Jamaica \\ ${ }^{2}$ Epidemiology Research Unit, Tropical Medicine Research Institute, University of the West Indies, Mona, Kingston 7, Jamaica \\ Correspondence should be addressed to Trevor Ferguson; trevor.ferguson02@uwimona.edu.jm
}

Received 26 October 2012; Accepted 17 December 2012

Academic Editor: John Kortbeek

Copyright (C) 2013 Tanya Sargeant et al. This is an open access article distributed under the Creative Commons Attribution License, which permits unrestricted use, distribution, and reproduction in any medium, provided the original work is properly cited.

\begin{abstract}
The medical literature contains only a few reports of rhabdomyolysis occurring in patients with dengue fever. We report the case of a 25-year-old Jamaican man who was admitted to a private hospital four days after the onset of an acute febrile illness with fever, myalgia, and generalized weakness. Dengue fever was confirmed with a positive test for the dengue antigen, nonstructural protein 1 . He remained well and was discharged on day 6 of his illness. On day 8 , he started to pass red urine and was subsequently admitted to the University Hospital of the West Indies. On admission he was found to have myoglobinuria and an elevated creatine phosphokinase (CPK) of 325,600 U/L, leading to a diagnosis of rhabdomyolysis. Dengue IgM was positive. He was treated with aggressive hydration and had close monitoring of his urine output, creatinine, and CPK levels. His hospital course was uneventful without the development of acute renal failure and he was discharged after 14 days in hospital, with a CPK level of $2463 \mathrm{U} / \mathrm{L}$. This case highlights that severe rhabdomyolysis may occur in patients with dengue fever and that early and aggressive treatment may prevent severe complications such as acute renal failure and death.
\end{abstract}

\section{Introduction}

Rhabdomyolysis is characterized by the rapid breakdown of skeletal muscle with leakage of muscle cell contents into the circulation $[1,2]$. These contents include electrolytes, myoglobin and other sarcoplasmic proteins, such as creatine kinase, lactate dehydrogenase, alanine aminotransferase, and aspartate aminotransferase [1]. The resulting myonecrosis presents clinically as limb weakness, myalgia and commonly, gross pigmenturia without haematuria $[1,2]$. Acute renal failure is a common complication of rhabdomyolysis and is due to the toxic effects of filtering excessive quantities of myoglobin in the setting of hypovolaemia [3]. The causes of rhabdomyolysis are protean, with acute viral infections such as influenza, HIV, coxsackievirus, and cytomegalovirus recognized as common causes $[1,4]$. Although the dengue virus shares several features with other viruses known to cause myopathies, dengue fever is not listed as a cause of rhabdomyolysis in major textbooks and review articles [4]. Over the last decade, a number of case reports of patients with dengue and rhabdomyolysis have been published in the literature [4-8]. We now report another case of rhabdomyolysis associated with dengue fever and present a literature review with a discussion of the clinical implications.

\section{Case Presentation}

A 25-year-old man with no known chronic illnesses presented to the University Hospital of the West Indies (UHWI) in Kingston, Jamaica, with a history of back pain and fever beginning eight days prior to his presentation. The fever lasted for two days and settled with the use of paracetamol. He then started to experience severe arthralgia, myalgia, and generalized muscle weakness which lasted for the first few days of his illness. He was seen by his primary care physician three days after the onset of symptoms and was admitted to a private hospital with a diagnosis of dengue fever. $\mathrm{He}$ reported that his platelet count at that time was $44,000 / \mu \mathrm{L}$ but remained asymptomatic, with no signs of bleeding, and 


\begin{tabular}{|c|c|c|c|c|c|c|c|}
\hline 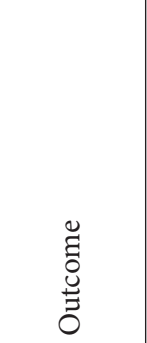 & 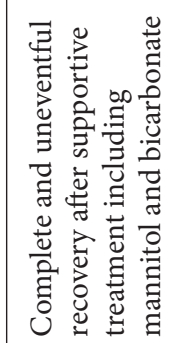 & 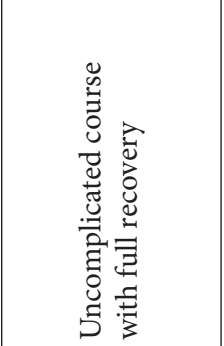 & 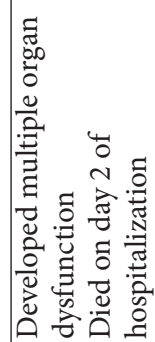 & 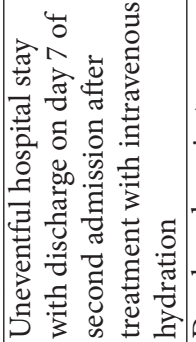 & 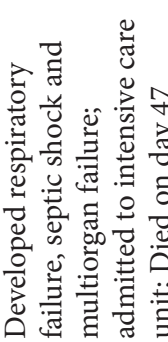 & 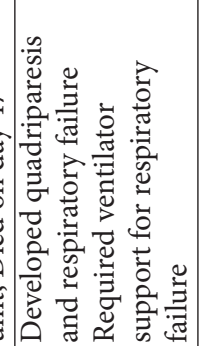 & 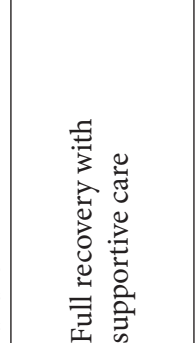 \\
\hline 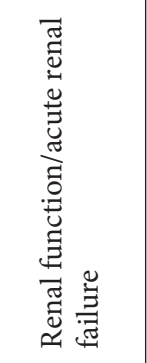 & 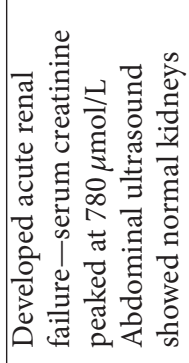 & 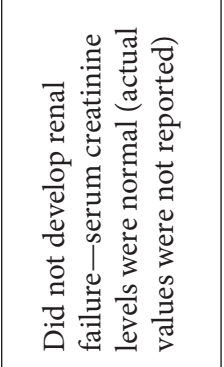 & 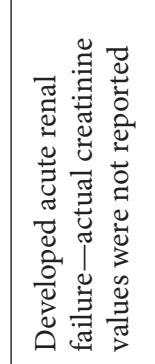 & 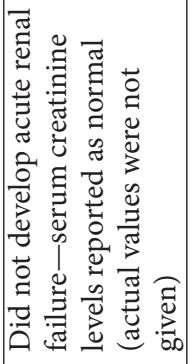 & 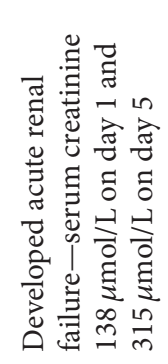 & 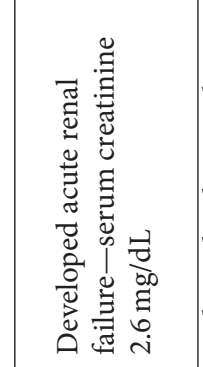 & 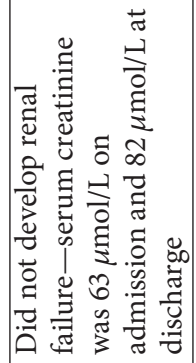 \\
\hline 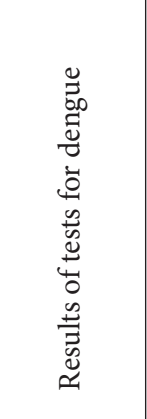 & 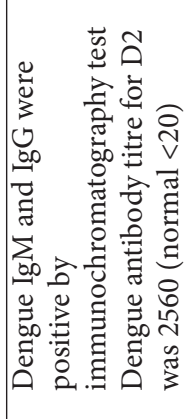 & 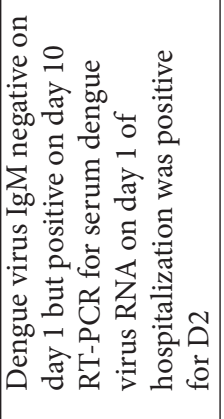 & 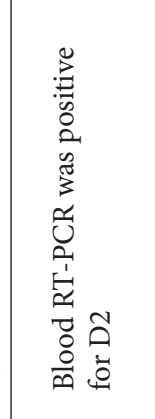 & 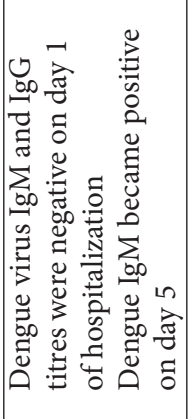 & 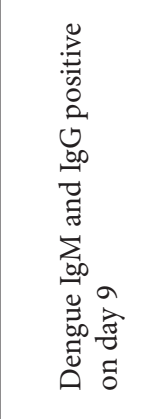 & 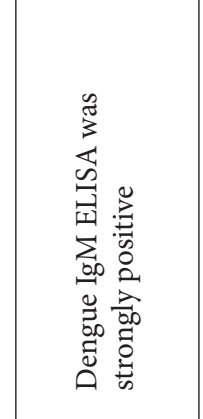 & 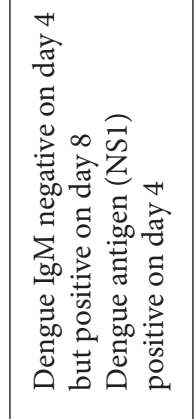 \\
\hline 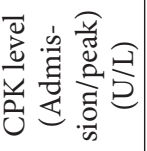 & 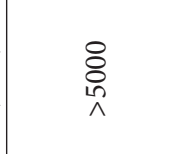 & 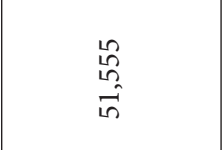 & 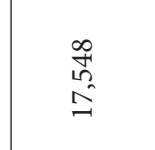 & $\begin{array}{l}\vec{\varnothing} \\
\circ \\
\infty \\
i n\end{array}$ & $\begin{array}{l}8 \\
\text { ఏे } \\
\text { டी }\end{array}$ & $\begin{array}{l}8 \\
\vdots \\
\hat{i}\end{array}$ & $\begin{array}{l}8 \\
0 \\
\text { in } \\
m\end{array}$ \\
\hline 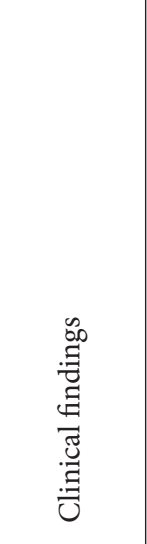 & 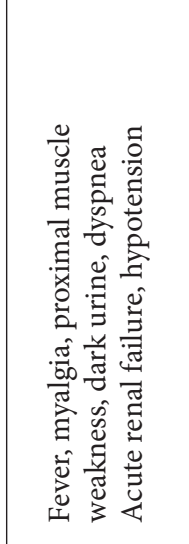 & 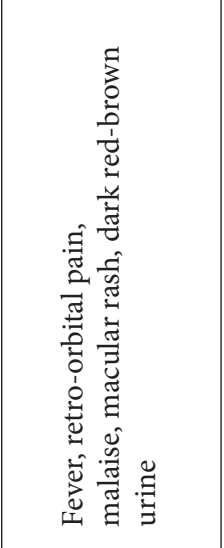 & 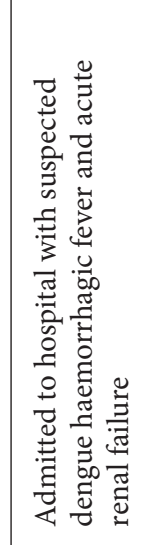 & 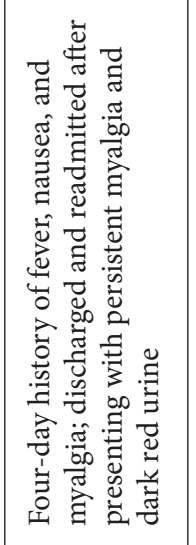 & 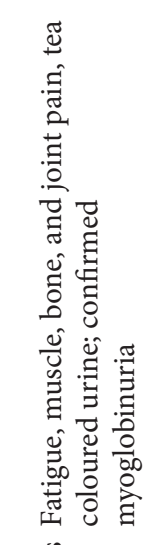 & 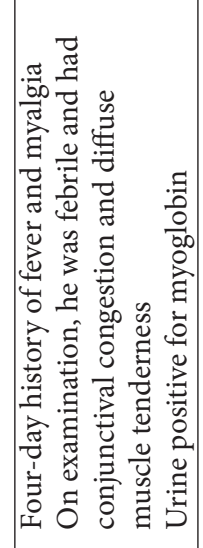 & 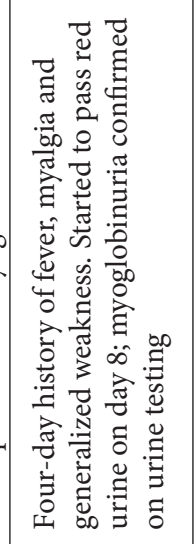 \\
\hline 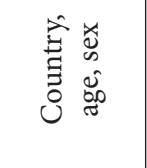 & 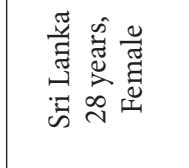 & 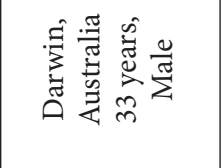 & 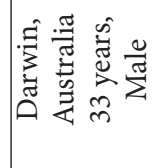 & 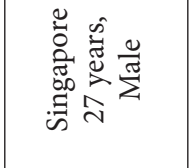 & 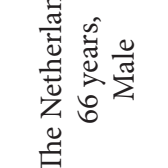 & 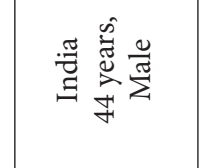 & 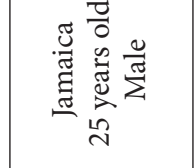 \\
\hline 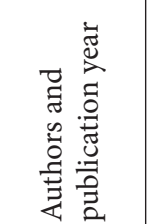 & 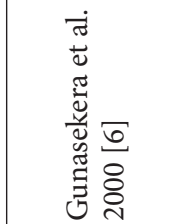 & 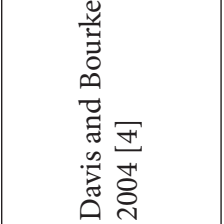 & 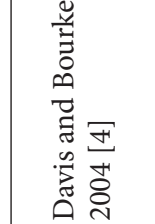 & 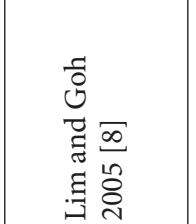 & 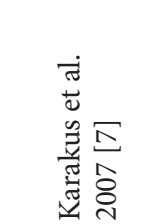 & 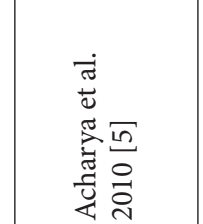 & 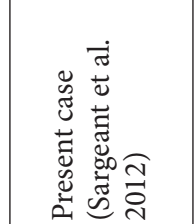 \\
\hline
\end{tabular}




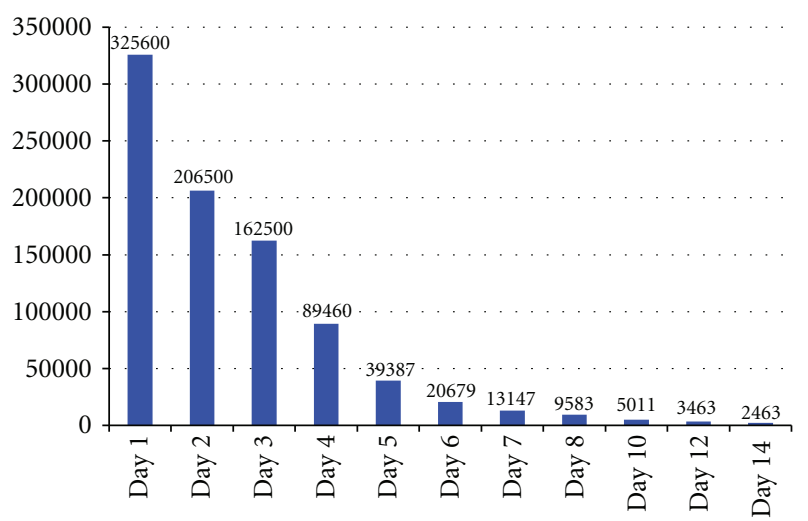

FIGURE 1: Trends in creatine phosphokinase (CPK) levels for case patient while in hospital.

was therefore discharged with a plan for followup as an outpatient. Two days later he noticed dark-coloured, then red urine and therefore returned to the private hospital and was subsequently referred to the UHWI for further management.

At the time of presentation, there were no reports of mucosal bleeding, skin haemorrhages, or haematochezia. There was no history of retro-orbital pain, neck stiffness, or photophobia. There were no other urinary symptoms and no history of nausea or vomiting. Of note, he gave no history of exposure to rats and he had not travelled recently. However, he did recall a neighbour having a diagnosis of dengue fever 1-2 weeks prior to the onset of his symptoms.

On physical examination, he had normal vital signs with a temperature of $36.3^{\circ} \mathrm{C}$. His cardiovascular, respiratory, abdominal, musculoskeletal, and central nervous systems were all normal. Examination of the skin did not reveal any petechiae, purpurae, ecchymoses, or rash. There were no wet purpurae in his mouth and fundoscopy was negative for retinal haemorrhages. A tourniquet test was performed and was negative. Urinalysis revealed a $\mathrm{pH}$ of 7.5 and tested positive for blood with no other abnormalities.

Investigations from the private hospital revealed an initial leukopaenia, neutropaenia, and thrombocytopaenia, verified by peripheral blood film. By the time of his presentation to the UHWI, his white blood cell count had normalized but the platelet count remained low at $49,000 / \mu \mathrm{L}$. However, manual review of the peripheral blood film revealed a higher count of $100,000 / \mu \mathrm{L}$. His initial urea, creatinine, electrolytes, and coagulation studies were normal and remained normal for the duration of his admission. Creatinine level was $63 \mu \mathrm{mol} / \mathrm{L}$ on admission and $82 \mu \mathrm{mol} / \mathrm{L}$ at discharge. Admission aspartate transaminase was $1841 \mathrm{U} / \mathrm{L}$ (normal 7-32 U/L), $\gamma$ glutamyl transferase was $159 \mathrm{U} / \mathrm{L}$ (normal 10-70 U/L), and lactate dehydrogenase was $5740 \mathrm{U} / \mathrm{L}$ (normal 105-200 U/L). These values declined steadily during the admission and eventually normalized by the time of discharge. His initial creatine phosphokinase (CPK) was 325,600 U/L (normal for a male $40-240 \mathrm{U} / \mathrm{L}$ ). With early and aggressive treatment, this value decreased by about $30-50 \%$ every day until it was $2,463 \mathrm{U} / \mathrm{L}$ at the time of discharge (see Figure 1). Urine microscopy was negative for red blood cells, casts, or other abnormal urinary sediments, but urinary myoglobin was positive. Tests for HIV, hepatitis B and C, and leptospirosis were all negative. Laboratory tests for the dengue virus on day four of his illness revealed a positive test for the dengue virus antigen, non-structural protein 1 (NS1), and negative immunoglobulin M (IgM). Dengue IgM became positive on day eight and remained positive on day 14 .

In-hospital management included aggressive hydration with intravenous and oral fluids, strict input/output charting, daily urinalyses to monitor $\mathrm{pH}$, and close monitoring of the urea, creatinine, electrolytes, $\mathrm{CPK}$, and platelet count. Target urine output was $200 \mathrm{~mL} /$ hour as recommended by Bosch and colleagues [1]. Urinary $\mathrm{pH}$ remained above 6.0 throughout and therefore alkalinization of the urine with sodium bicarbonate was not instituted. The patient's hospital course was uncomplicated, he remained clinically well and was discharged with instructions to return initially for review on the ward and then for followup in the out-patient clinic.

\section{Discussion}

This patient fulfils the criteria for a confirmed case of dengue fever as defined by the World Health Organization (WHO) [9] having presented with an acute febrile illness associated with headache, myalgia, arthralgia and leukopaenia and occurring at the same location and time as another confirmed case of dengue fever. It was verified by a positive IgM antibody test on the late acute and convalescent serum specimens and there was also demonstration of dengue virus antigen (NS1) in his serum. His was also a case of rhabdomyolysis with the typical triad of generalized weakness, myalgia, and dark urine/myoglobinuria associated with a CPK that was more than five times the upper limit of normal [2]. Although there are case reports of rhabdomyolysis associated with dengue fever, major textbooks do not mention the dengue virus as a possible cause of rhabdomyolysis [4, 7]. We found only one review article on atypical manifestations of dengue fever which includes rhabdomyolysis as a possible complication [10].

A review of the published literature using the PubMed database identified five case reports of rhabdomyolysis associated with dengue fever [4-8]. The findings of the six patients in these case reports and the present case are summarized in Table 1. In each case, there was confirmation of both dengue fever and rhabdomyolysis. Of note six of the seven cases were males, with ages ranging from 25 to 66 years; the lone female was 28 years old. CPK levels ranged from 5,000 to $325,600 \mathrm{U} / \mathrm{L}$. Two of the seven patients died (29\%) while four of them developed acute renal failure (57\%) and two patients developed respiratory failure (29\%). These clinical findings suggest that rhabdomyolysis in patients with dengue carries high morbidity and mortality rates when compared to the $8 \%$ overall mortality and $13-50 \%$ incidence of acute renal failure for all patients with rhabdomyolysis $[1,2]$. In the cases presented, mortality was associated with the presence of acute renal failure and/or multiorgan failure.

The mechanisms involved in the development of rhabdomyolysis in patients with dengue fever are unknown [4]. 
However, rhabdomyolysis is reported to occur in a number of viral infections, such as influenza $A$ and $B$, coxsackievirus, Epstein-Barr virus, and HIV [1]. Davis and Bourke suggest that since the dengue virus shares several features with these other viruses known to cause severe myositis, it is not surprising that dengue could also cause rhabdomyolysis [4]. They further suggest that the most likely cause may be due to myotoxic cytokines, particularly tumour necrosis factor (TNF) and interferon alpha (IFN- $\alpha$ ) released in response to a viral infection [4]. Muscle biopsy specimens from patients with acute viral myositis have revealed a range of findings, from a mild lymphocytic infiltrate to foci of severe myonecrosis but direct invasion of muscle by the virus has not been consistently demonstrated [4].

Acute kidney injury associated with myoglobinuria is the most serious complication of both traumatic and nontraumatic rhabdomyolysis and may be life-threatening. As seen in the cases reviewed, acute renal failure is a frequent complication in patients with dengue and rhabdomyolysis. The exact mechanisms by which rhabdomyolysis impairs renal function are unclear, but experimental evidence suggests that intrarenal vasoconstriction, direct and ischaemic tubular injury, and tubular obstruction all play a role [1]. Myoglobin becomes concentrated along the renal tubules, where it precipitates when it interacts with the TammHorsfall protein, particularly in the presence of acidic urine. Myoglobin seems to have no marked nephrotoxic effect in the tubules unless the urine is acidic, hence the common practice of urinary alkalinization as part of supportive treatment measures.

There is no specific threshold value of serum CPK above which the risk of acute kidney injury is markedly increased, but the risk is usually low when CPK levels at admission are less than 15,000-20,000 U/L. Acute kidney injury has been reported with CPK values as low as $5000 \mathrm{U} / \mathrm{L}$, but this usually occurs with coexisting conditions such as sepsis, dehydration, and acidosis [1]. In the published case reports of dengue and rhabdomyolysis, CPK levels ranged from a low of 5,000 U/L to a high of $156,000 \mathrm{U} / \mathrm{L}$. Our patient had an even higher CPK level of 325,600 U/L but did not develop acute renal failure.

\section{Conclusion}

Rhabdomyolysis should be recognized as a possible complication of dengue fever and should be reflected as such in medical textbooks especially in light of the possibility of acute renal failure and high mortality. Although causation has not been unequivocally established, supporting evidence for a causal association is the fact that rhabdomyolysis occurs commonly with influenza and other viruses with which the dengue virus shares many similarities.

Clinicians should note that the presentation of rhabdomyolysis can be subtle but its complications, in particular, acute kidney injury and multiorgan failure, can be devastating. These adverse effects are preventable with early recognition and institution of the appropriate management. We agree with Davis and Bourke that all patients with dengue fever should have a urinalysis done and that those who test positive for blood should have urine microscopy and a CPK test in order to determine if the patient may have rhabdomyolysis. This approach could be potentially lifesaving.

\section{Acknowledgments}

The authors wish to thank the patient who kindly gave consent for his case to be presented in this paper. We also thank the Epidemiology Research Unit (Tropical Medicine Research Institute) and the University Hospital of the West Indies for institutional support.

\section{References}

[1] X. Bosch, E. Poch, and J. M. Grau, "Rhabdomyolysis and acute kidney injury," New England Journal of Medicine, vol. 361, no. 1, pp. 62-72, 2009.

[2] G. Cervellin, I. Comelli, and G. Lippi, "Rhabdomyolysis: historical background, clinical, diagnostic and therapeutic features," Clinical Chemistry and Laboratory Medicine, vol. 48, no. 6, pp. 749-756, 2010.

[3] D. B. Hellmann and J. B. Imboden Jr., "Musculoskeletal \& immunologic disorders," in CURRENT Medical Diagnosis \& Treatment, M. A. Papadakis, S. J. McPhee, and M. W. Rabow, Eds., chapter 20, McGraw-Hill, New York, NY, USA, 2013.

[4] J. S. Davis and P. Bourke, "Rhabdomyolysis associated with dengue virus infection," Clinical Infectious Diseases, vol. 38, no. 10, pp. e109-e111, 2004.

[5] S. Acharya, S. Shukla, S. N. Mahajan, and S. K. Diwan, "Acute dengue myositis with rhabdomyolysis and acute renal failure," Annals of Indian Academy of Neurology, vol. 13, no. 3, pp. 221-222, 2010.

[6] H. H. Gunasekera, A. V. Adikaram, C. A. Herath, and H. H. Samarasinghe, "Myoglobinuric acute renal failure following dengue viral infection," The Ceylon Medical Journal, vol. 45, no. 4, p. $181,2000$.

[7] A. Karakus, N. Banga, G. P. Voorn, and A. J. Meinders, "Dengue shock syndrome and rhabdomyolysis," Netherlands Journal of Medicine, vol. 65, no. 2, pp. 78-81, 2007.

[8] M. Lim and H. K. Goh, "Rhabdomyolysis following dengue virus infection," Singapore Medical Journal, vol. 46, no. 11, pp. 645-646, 2005.

[9] M. G. Teixeira and M. L. Barreto, "Diagnosis and management of dengue," British Medical Journal, vol. 339, p. b4338, 2009.

[10] S. Gulati and A. Maheshwari, "Atypical manifestations of dengue," Tropical Medicine and International Health, vol. 12, no. 9, pp. 1087-1095, 2007. 


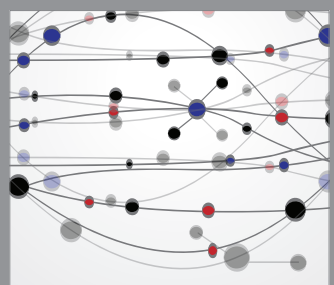

The Scientific World Journal
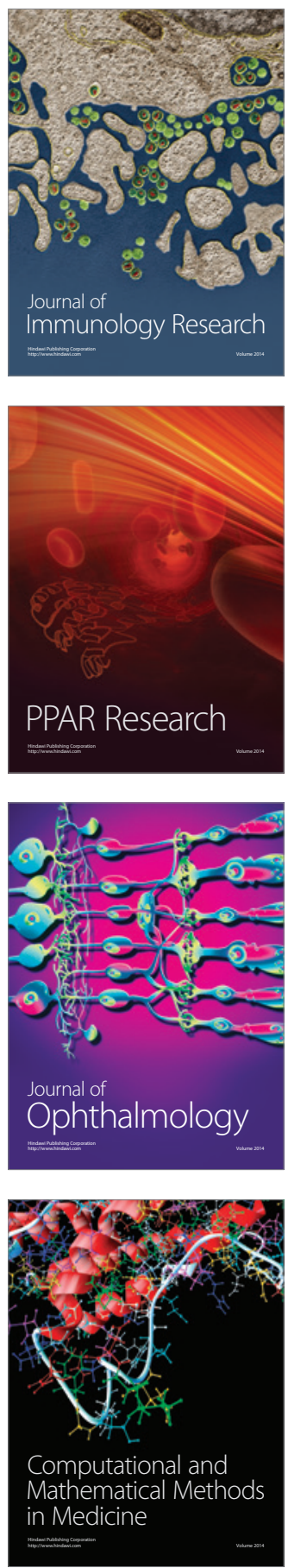

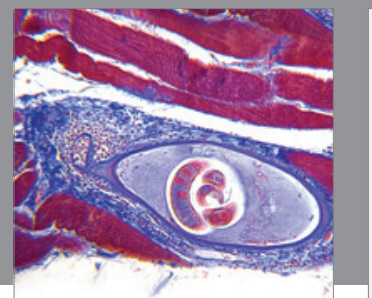

Gastroenterology

Research and Practice
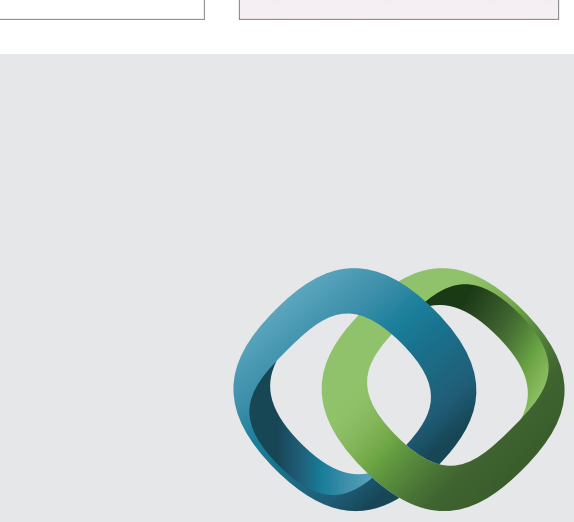

\section{Hindawi}

Submit your manuscripts at

http://www.hindawi.com
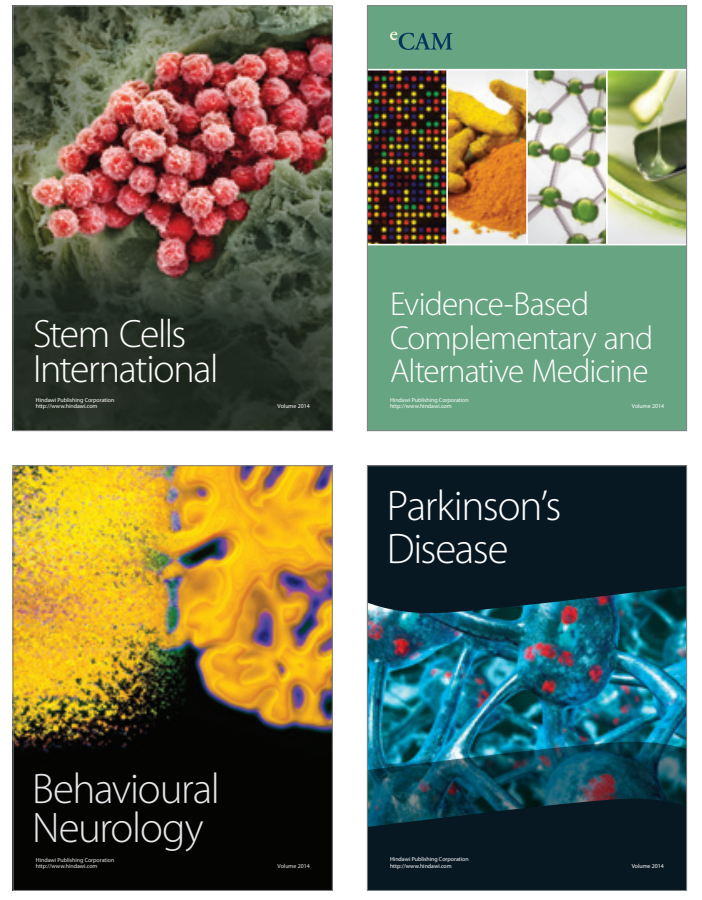
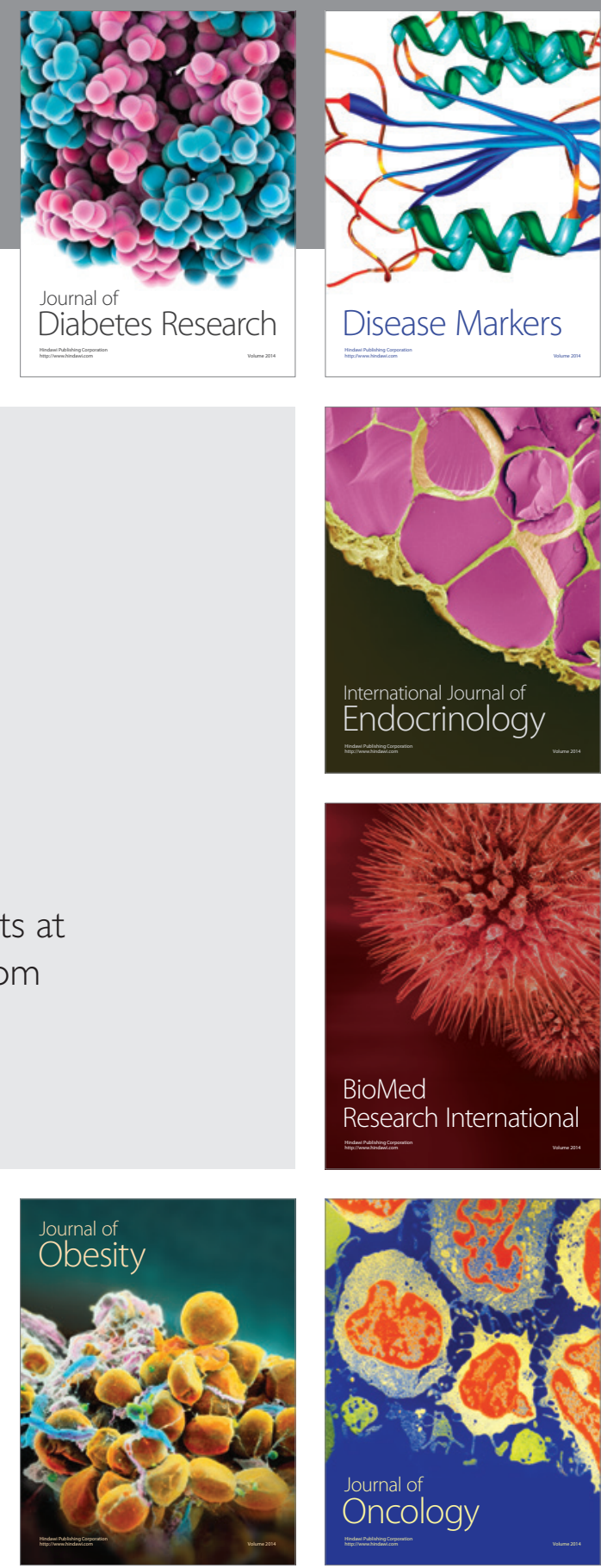

Disease Markers
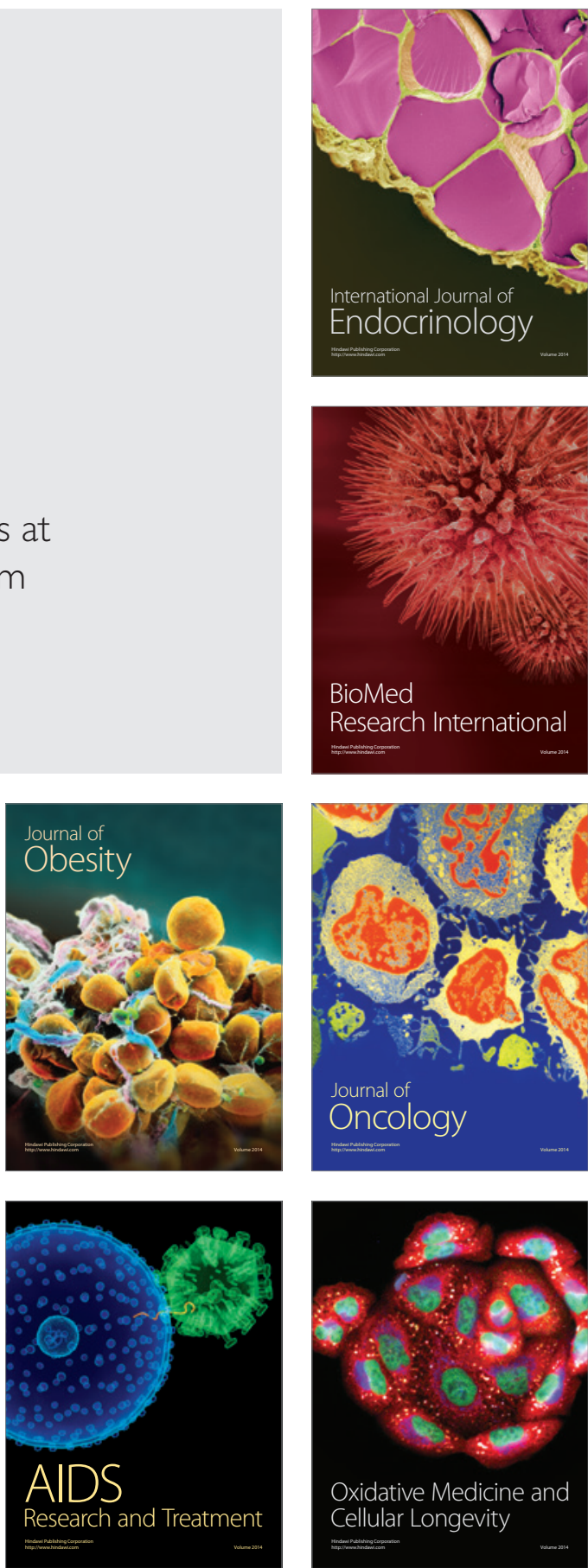